\section{PL-7 Positive Antisynthetase Syndrome and Pulmonary Hypertension}

To the Editor:

Antisynthetase syndrome is characterized by interstitial lung disease (ILD), myositis, arthropathy, fever, Raynaud phenomenon, and mechanic's hands. The morbidity of the syndrome is usually due to lung involvement ${ }^{1}$.

In January 2005, a 65-year-old female nonsmoking patient presented to our outpatient clinic with fever $\left(38.5^{\circ} \mathrm{C}\right)$, unproductive cough, shortness of breath, dry throat, nocturnal sweating, and weight loss (3-4 kg). Previously, she was exposed to passive cigarette smoke and cleaning chemicals. Essential hypertension treated by bisoprolol and an allergy to contrast material were known. There was no positive family history for rheumatic or cardiovascular disease. Electrocardiogram was normal but lung function test showed a restrictive pattern [forced vital capacity (FVC) $52 \%$, forced expiratory volume (FEV)1 56.3\%, FEV1/FVC 85.1\%]. Carbon monoxide diffusion capacity measurement was not feasible because of the decreased vital capacity. Glomerular filtration rate was slightly reduced $(48 \mathrm{ml} / \mathrm{min})$. Other variables were elevated: C-reactive protein $18 \mathrm{mg} / \mathrm{l}$ (normal $<5$ $\mathrm{mg} / \mathrm{dl}$ ), creatine kinase (CK) $1402 \mathrm{U} / \mathrm{l}, \mathrm{CK}$-myocardial band $45 \mathrm{U} / \mathrm{l}$, lactate dehydrogenase $335 \mathrm{U} / 1$, and pro-brain natriuretic peptide (NT-proBNP) $426 \mathrm{pg} / \mathrm{ml}$. Antinuclear antibodies (ANA) were negative. Chest radiograph showed cardiomegaly and pulmonary reticulonodular pattern (Figure 1A). Chest thin-slice computed tomography (CT) detected ground-glass opacities and signs of fibrosis (Figure 2). Bronchoalveolar lavage revealed normal cell numbers but echocardiography showed elevated systolic pulmonary arterial pressure $(42+10 \mathrm{mmHg})$. The absence of signs of alveolitis in the bronchoalveolar lavage in the presence of ILD with severe fibrosis and ground-glass opacities in the CT may be surprising, but such discrepancies are quite common in collagen vascular diseases. The patient was given longterm oxygen therapy $(1 \mathrm{l} / \mathrm{min})$, spironolactone $(25 \mathrm{mg})$, furosemide (30 mg), and prednisolone $(25 \mathrm{mg})$. Because of elevated total airway resistance $(0.5 \mathrm{kPa} \times \mathrm{s} / \mathrm{l})$, inhaled tiotropium was added to the therapeutic scheme despite limited benefit. After 2 months the patient felt better. The dry cough had improved and CK was in the normal range, but she was still complaining of shortness of breath during exercise. Right heart catheterization showed a mildly elevated mean pulmonary arterial pressure (mPAP) and resistance [mPAP $22 \mathrm{mmHg}$; pulmonary vascular resistance (PVR) 319 dyn s cm${ }^{-5}$, cardiac index (CI) $2.61 \mathrm{l} / \mathrm{min} / \mathrm{min}^{2}$ ] with no signs of left ventricular dysfunction [pulmonary arterial wedge pressure (PAWP) $5 \mathrm{mmHg}$.

During the next 4 years, the patient was treated with acetylcysteine (600 mg TID), spironolactone (50 mg), cyclosporine (50 mg), prednisolone
(20 mg every other day), longterm oxygen therapy, and physiotherapy. After the fourth year of followup the patient presented with arthritis urica, which was limited to the first digit of the right foot. Gaenslen sign was positive, but there were no other signs of arthritis or Raynaud phenomenon. Pulmonary embolism was ruled out by $\mathrm{CT}$ angiography and ventilation-perfusion scan. CK was in the normal range, and radiograph (Figure 1B), pulmonary function tests, lung scintigraphy, and polysomnography were unchanged while pulmonary hemodynamics were significantly deteriorated and right heart catheterization confirmed manifest pulmonary hypertension (PH) associated with lung fibrosis (mPAP $34 \mathrm{mmHg}$, PVR 415 dyn s cm${ }^{-5}$, PAWP $11 \mathrm{mmHg}$, CI $2.57 \mathrm{l} / \mathrm{min} / \mathrm{m}^{2}$ ). The detection of antiPL-7 (antithreonyl-tRNA synthetase) antibodies positivity prompted the diagnosis of antisynthetase syndrome with ILD and PH. Treatment with sildenafil $20 \mathrm{mg}$ TID was started while other medication remained unchanged. During 2 years of followup, PL-7 testing remained positive, exercise capacity improved, NT-proBNP values returned to normal, and lung function test remained unchanged (FVC 52.6\%, FEV1 49\%, FEV1/FVC 91.2\%, $\left.\mathrm{pO}_{2} 73.1 \mathrm{mmHg}, \mathrm{pCO}_{2} 34.6 \mathrm{mmHg}, \mathrm{SO}_{2} 96.7 \%\right)$. The patient was tested positive for PL-7; however, cytoplasmic fluorescence was not present.

To our knowledge, this is the first report of PL-7-positive antisynthetase syndrome with ILD and PH. The delay of the diagnosis was significant because the PL-7 antibodies were evidenced only 4 years after diagnosis of ILD.

The importance of PL-7 antibodies in myositis has been recognized for more than 2 decades $^{2}$. A suspicion of antisynthetase syndrome should be raised when patients are presenting with ILD and negative ANA but increased CK or aldolase $^{3}$. A recent analysis of Jo-1 vs PL-7 and PL-12-positive antisynthetase syndromes found that PL-7 and PL-12 patients had a very similar phenotype characterized by more ILD and less myositis as compared to Jo- 1 syndrome ${ }^{4}$. PL-7 and PL-12 patients were more prone to dyspnea and poor survival when compared to anti Jo-1-positive patients ${ }^{5}$. Pericarditis is also a possible manifestation related to anti-PL-7 antibodies ${ }^{6}$, but in our patient there was no evidence of pericarditis. Recently, a retrospective analysis of 12 PL-7-positive patients identified 3 with echocardiographic signs of pulmonary hypertension. Unfortunately, right heart catheterization was not performed and therefore left ventricular involvement could not be ruled out ${ }^{7}$. A retrospective analysis of a considerable number of patients with antisynthetase syndrome showed that the presence of pulmonary hypertension is associated with decreased survival ${ }^{8}$.

Pulmonary involvement predicted good response for diseasemodifying antirheumatic drug use in patients with anti Jo- $1^{1}$ but it remains
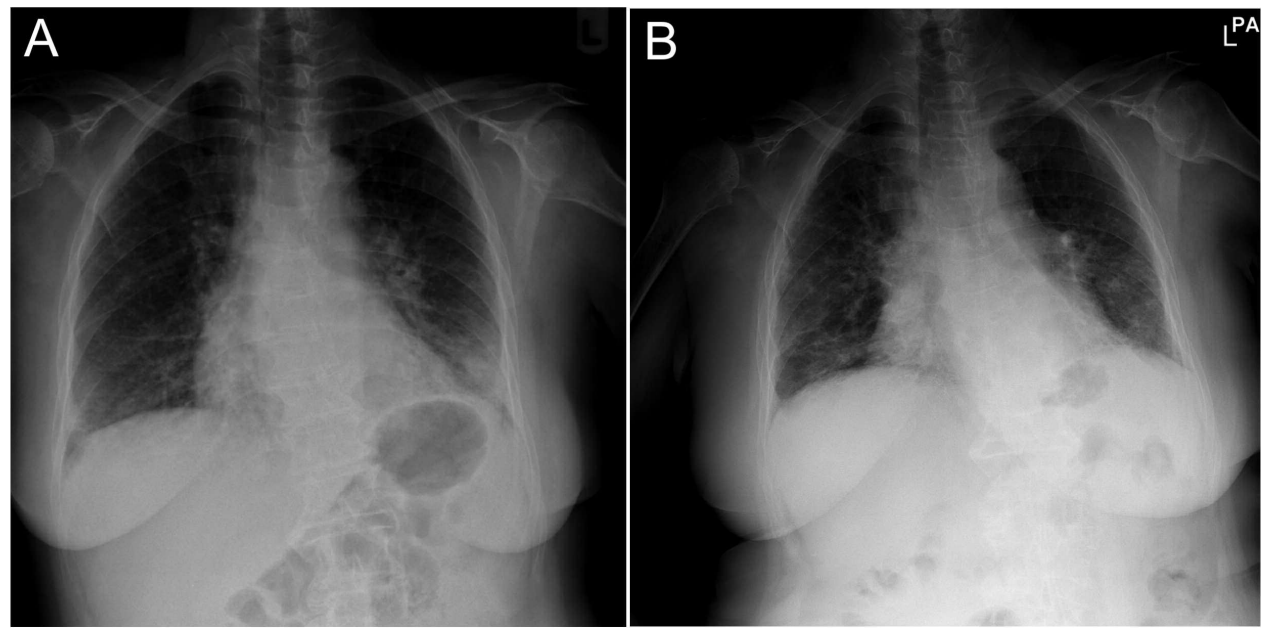

Figure 1. A. Chest radiograph shows cardiomegaly and pulmonary reticulonodular pattern. B. Followup chest radiograph after 4 years is unchanged. 


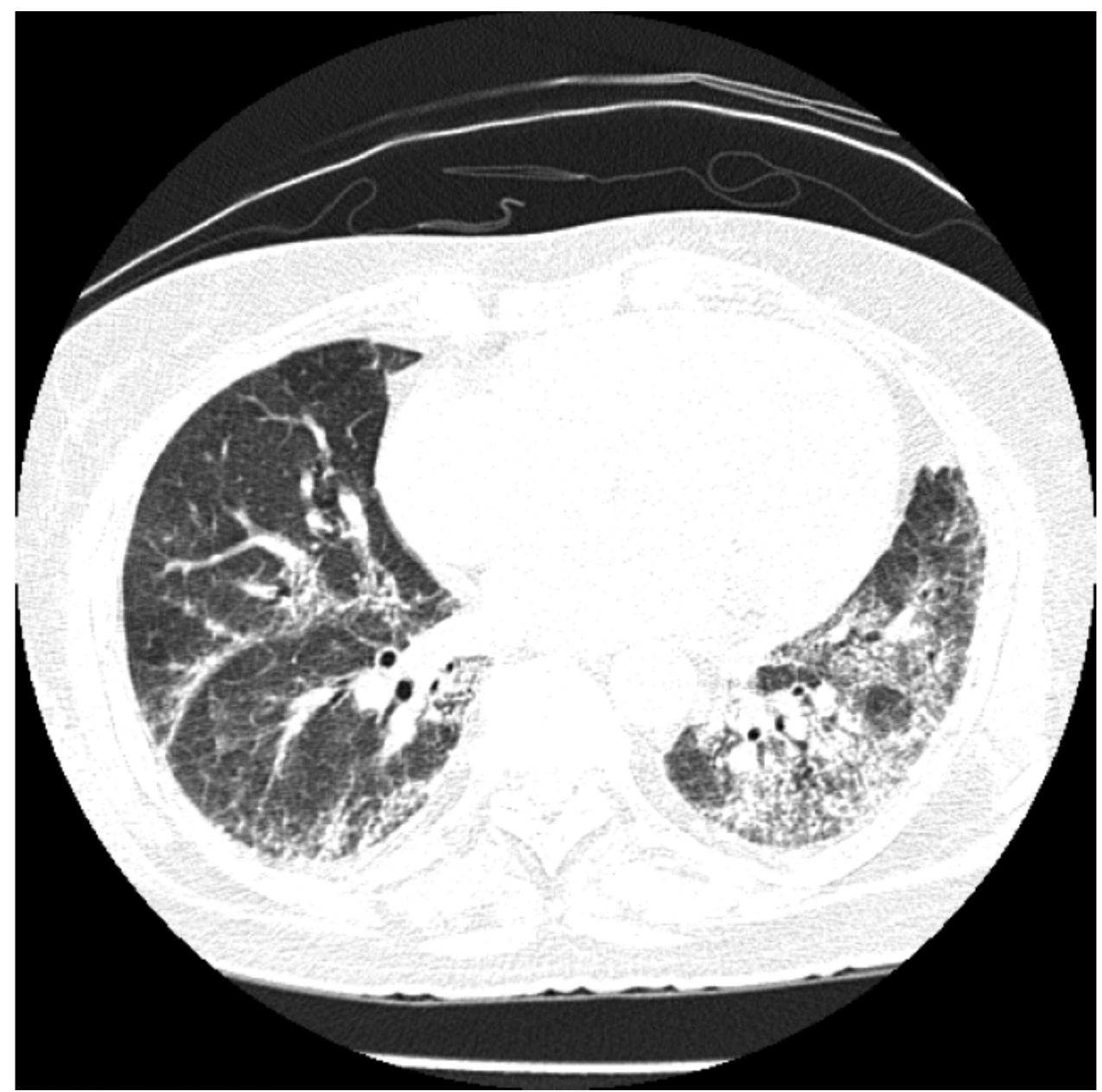

Figure 2. Chest thin slice computed tomography detected ground-glass opacities and signs of fibrosis.

speculative whether the same applies for patients with anti-PL-7 antibodies. Our case suggests that the combination of cyclosporine and corticosteroid with high-dose acetylcysteine may have contributed to control of progression of ILD but not of PH.

Our findings on PH in a PL-7-positive antisynthetase syndrome suggest that immunosuppressive treatment may control ILD but not pulmonary vascular involvement.

\section{ACKNOWLEDGMENT}

We thank Professor Ulrike Demel, Medical University of Graz, Division of Rheumatology, for repeated laboratory assessment of PL-7 antibodies and Professor Andrea Olschewski, Ludwig Boltzmann Institute for Lung Vascular Research, for critical discussion of the manuscript.

VASILE FORIS, MD; GABOR KOVACS, MD, Ludwig Boltzmann Institute for Lung Vascular Research, and Medical University of Graz, Department of Internal Medicine, Division of Pulmonology, Graz, Austria; MARCO MATUCCI-CERINIC, MD, PhD, Department of Biomedicine, Division of Rheumatology, AOUC and Department of Experimental and Clinical Medicine, University of Florence, Florence, Italy; HORST OLSCHEWSKI, MD, PhD, Ludwig Boltzmann Institute for Lung Vascular Research, and Medical University of Graz, Department of Internal Medicine, Division of Pulmonology. Address correspondence to Dr. H. Olschewski, Medical University of Graz, Department of Internal Medicine, Division of Pulmonology, A-8036 Graz, Auenbruggerplatz 20, Austria. E-mail: horst.olschewski@klinikum-graz.at.

\section{REFERENCES}

1. Stanciu R, Guiguet M, Musset L, Touitou D, Beigelman C, Rigolet A, et al. Antisynthetase syndrome with anti-Jo1 antibodies in 48 patients: pulmonary involvement predicts disease-modifying antirheumatic drug use. J Rheumatol 2012;39:1835-9.

2. Mathews MB, Reichlin M, Hughes GR, Bernstein RM. Anti-threonyl-tRNA synthetase, a second myositis-related autoantibody. J Exp Med 1984;160:420-34.

3. Fischer A, Swigris JJ, Du Bois RM, Lynch DA, Downey GP, Cosgrove GP, et al. Anti-synthetase syndrome in ANA and anti-Jo-1 negative patients presenting with idiopathic interstitial pneumonia. Respir Med 2009;103:1719-24.

4. Hervier B, Devilliers H, Stanciu R, Meyer A, Uzunhan Y, Masseau A, et al. Hierarchical cluster and survival analyses of antisynthetase syndrome: phenotype and outcome are correlated with anti-tRNA synthetase antibody specificity. Autoimmun Rev 2012;12:210-7.

5. Marie I, Josse S, Decaux O, Dominique S, Diot E, Landron C, et al. Comparison of long-term outcome between anti-Jo1- and anti-PL7/PL12 positive patients with antisynthetase syndrome. Autoimmun Rev 2012;11:739-45

6. Labirua-Iturburu A, Selva-O'Callaghan A, Vincze M, Dankó K, Vencovsky J, Fisher B, et al. Anti-PL-7 (anti-threonyl-tRNA synthetase) antisynthetase syndrome: clinical manifestations in a series of patients from a European multicenter study (EUMYONET) and review of the literature. Medicine 2012;91:206-11. 
7. Hervier B, Uzunhan Y, Hachulla E, Benveniste O, Nunes H, Delaval P, et al. Antisynthetase syndrome positive for anti-threonyl-tRNA synthetase (anti-PL7) antibodies. Eur Respir J 2011;37:714-7.

8. Hervier B, Meyer A, Dieval C, Uzunhan Y, Devilliers H, Launay $\mathrm{D}$, et al. Pulmonary hypertension in antisynthetase syndrome: prevalence, etiology and survival. Eur Respir J 2013; Feb 8 (E-pub ahead of print).

J Rheumatol 2013;40:10; doi:10.3899/jrheum.130403 